\title{
RELIGIONES DFL EXTREMO ORJENTE
}

Antonio Bentue

Vamos a trator sobre la familia religiosa del Extremo Orien te, vinculada al Hinduismo.

guire tres puntos:

Distribuire el tena en un pequeño preliminar y luego distin

1. La religión hindó en su forma más antigua o védica (de "Vedas", que son los grandes textos sagrados del vedismol.

2. La segunda tarea serb la elaboración, desde ese punto de partida, de lo que se denomino el Hinduismo clisico. Todo esto surgib y se desarrollo alrededor de 1.800 a 1.400 a.C... respecto de la formación de la religión védica y en el siglo VIII al VI a.C. en cuanto al Hin duismo clésico.

3. Finalmente, $e l$ siglo $V I$ a.C. se caracteriza por una gran riqueza religiosa en todo el mundo, no solo en la India, sino también en Irón, en la tradición bíblica, en China mismo. Es en este siglo cuando se producen en el Hinduismo clasico rupturas que dan origen al Budismo -que es la ruptura principal-, al Jainismo (cuyas tradi ciones precursoras se remontarian a mas de dos siglos ontesl y al Krishnaismo, aún cuando la aparición del culto a Krishno se habría producido antes.

\section{PRELIMINAR}

Antes de comenzar querría aclarar dos cosas: En primer lu -

gar, que todo este movimiento tan lejano a nosotros responde en el fondo a lo mismo búsquada religiosa que es la nuestra, en nuestra propia tradición. En el fondo, pienso que cuando uno enfoco un tipo de reli gión lejana tiene que superar dos prejuicios: Uno, ver eso religion y la propia como en un mismo saco; es decir, que da lo mismo ser budista - ser cristiano. El prejuicio opuesto es despreciarla, verla un poco co mo un folklore, o una cosa lejana, ccmo un "accidente" de las búsquedas humanas al margen de la verdad que uno posee. Son esos dos extremos que hay que evitar.

Pora lograrlo hay que tener siempre presente que lo reli gib́n, sin agotorse en esto perspectivo, consiste en lo siguiente: lo 
Realidad, que llamamos el Absoluto, Díos, es una y por tanto, desde el punto de vista de la realidad "objetiva" buscada por cualquier creyen te, es exactamente la misma. Aquello que los musulmanes quieren de = cir con Alb, es lo mismo que los judíos quieren decir con Yaweh, lo mismo que los cristianos quieren decir con Dios Padre, y 10 mismo que los budistas pretenden decir con Nirvana, o los hinduistas con Brahma. Es la misma y exacta realidad. A nivel de la realidad "objetiva" Dios es uno, y solo uno, $y$ en ese sentido todo fo tiene por objeto la misma y única realidad Absoluta. Si es asf, todos los creyentes en el fondo creemos en $10 \mathrm{mismo.} \mathrm{El} \mathrm{objeto} \mathrm{de} \mathrm{nuestra} \mathrm{fe} \mathrm{es} \mathrm{exuctamente} \mathrm{el} \mathrm{mis} \mathrm{-}$ mo.

Otra cosa son las mediaciones humanas de la cultura con las cuales los diferentes grupos humanos ubicados en diversos lugares $y$ en diversos tiempos, y por lo tanto condicionados por sus propias tradiciones, y hasta por el entorno geografico dan forma a esa única Realidad. El lenguaje con que en esos diversos medios se intenta expre sar esa única realidad, cambio, es diferente, esto condicionado por el tiempo y por el lugar distinto en que cada grupo humano busca fundar en lo Absoluto la propia realidad no fundada.

En ese sentido yo diría lo siguiente: aqui, en este lado do la línea estamos los hombres, estb la historia humana, con su tiempo y su espacio. Todos los hombres estamos desde nuestra diferente ubicación, temporal y espacial, intentardo "decir" la realidad indecible, acceder a esa realidad inaccesible, que es Dios. Por io tanto, yo enunciarla una formula: "Io subjetivamente distinto, objetivamente es igual". El objeto de toda tradición religiosa es el mismo, aunque las mediaciones humanas y por tanto los lenguajes, las imágenos de ese Absoluto, las maneras de decirlo, de formularlo, varian de acuerdo a tradiciones, culturas, etc.

Esto podria conducir diractamente a la trampa del primer prejuicio anteriormente expresado: ya que en el fondo todo lenguaje hu mono sobre Dios dice "objetivamente" $10 \mathrm{mismo,} \mathrm{sólo} \mathrm{que} \mathrm{cada} \mathrm{uno} \mathrm{se} \mathrm{-}$ gún su propio condicionamiento, da 10 mismo cualquier religión. En par te esto es verdad. La misma Iglesia Catblica lo reconoce en el Vati cano II, al abrirse y establecer contacto oficial con las religiones no cristianas; incluso un documento conciliar estó dedicado al tema. $Y$ el tratamiento que hace es justamente reconocer que en las demas religiones hay verdad.

¿̇b6nde esta la posibilidad de superar esa primera Impre sión de que todas las religiones caben en el mismo sacó? Esto en lo si guiente, según mi parecer: en que Dios es inaccesible por definición." Es lo que no es el hombre, lo que no es el murdo, el Otro, la Trascenm dencia. Y por lo tanto el hombre, en la inmanencia, por mucho que ha ga, nunca va a poder acceder por su cuenta a esa Trascendencia. Es inaccesible... a no ser que Dios establezca un mediador, es decir, un puente de acceso. Y el puente de acceso es lo que en nomenclatura re- 
ligiosa podrfamos llamar la revelacien de Dios. Y sobre esto se pue de establecer comparación; se pueden eomparar las diversas formas de revelución: Todas las religiones pretenden tener una revelación dada por Dios, consignade en "Sngrodus Eserituras" al estilo de la nues tra. El Corón es "Sagrada Escricura" para los musulmanes; los Vedas lo son para los hindues, ete. For tanto, los diversas religienes tienen la pretensión de poseer un documento recibido, no inventado por el hombre. Y asi tenemus que la diversidad de revelcciones permite comparar entre elles y establecar objetivemente una especie de jerarquización ccerca de la mayor o mener cercanfa a lograr lo que se pretende: el cicceso a Dios.

Entonces éque criterios existen para que uno pueda conocer la validez de su opeión por la religión a que pertenece? \&Y ello más allá del condicionamiento de la propia tradición religiosa?

Se debe tomar en cuenta, también, que si se plensa hones tamente, con cierta sensatez, serfo razonable sospechar, por ejem plo, que la persona que aquí es un fiel cristiano, si hubiera nacido de padres hindúes y viviera en la India, con mucha posibilidad serla un hinduista con la misma seguridad y convicción con los que acó as un catblico (dejando aparte conversiones "extras", como los que hay tambien en el hinduismol. Son condicionamientos que uno puede pre ver y son cosas para considerar en sesio y tomar distancia de la sub jetividad de cada uno de elles.... Ahoro bien, repitiendo la preguñ ta, écóno saber quévalidez objetiva tiene la propia opciśn?

Por un lado, yo contesturta: Mi opción tiene validez objetiva, sea cual seo. Por lo tarito, en mi opción crietiona, en el forido, no tengo nucio -en lo fundamentel- que busear en la tradi ción corínica (islämica) que no encuentro en el cristicnismo. L.o que, en principio podría decirse rambién por parte de un musulmán o de un hindú. Es decir, mi búsqueda religiosa, condicionado por un lenguaje, por una trodición humana, toca una verdod objetiva, como minimo iqual a la que tocran las búsquedas de otres trudiciones. Por tanto, nodie esté "por debajo". Si alguien preguntara: "Yo esioy du dando si me hago budista", habrla que decirle: "Para te, ser un buen cristiano es de entrada lo mismo que ser un buen budista".

Entonces, la pregunta es otra: ése pueda llegar a mas. objetivanente hablandoß Yo diría, ese mís dependerá de cómo $\in \mathbf{I}$ puente de acceso, -la revelación que llega al hombre y que le permite acceder a Dios-, puede mostrarse más genial en el sentido siguien te: ¿́cuál es una respuesta abjetivamente mós adecuada para el pro blema que plantea la existericia del hombre?

En la medida en que yo pueda mostrar que el problema de la existencia tiene tal envergadura y que la geniulidad que pretende solucionor ese problema, con una revelocibn scivffica que acierta mejor siendo algo así como un mejor botín que otro pora ese determina- 
do ojal, verifico lo que es verificoble de una mayor cercanía entre nuestro lenguaje religioso, condicionado, por el cual pretendemos ac ceder a Dios y el acceso dujetivo que Dios haya podido establecer en tre su realidad y lo nuestru. Porque si Dios quiere salvar al hombre, con un puente es suficiente, no necesita medjadores. Los cris tianos creemus que ese puente es Cristo. En esa hipotesis, el cristiano cree que no sólo se salvan les cristicnos, sino que todos los que se salvan, subjetivarente confiados en su propia opuisn condi cionada por sus diversas tradiciones, objerivomente se salvan por el unico mediador, Cristo, que es el establecido objetivamente por Dios.

Quise simplemente mostrar, como preliminar, lo que me pa rece importante como actitud cuardio uno enfoca estos problemas, para superar los prejuicios iniciales. El prejuicio de que "todo da lo mismo porque es un mismo todo". En parie es verdad el "prejuicio" -como lo plantea el Vaticano II-, de reconocer que la verdad objetiva, Dios, es la misma en cualquier religión, con cualquier nombre co mo se le llame. Buscamos el fundamento de nuestra existencia, que se 1 lama Dios. $Y$ en esa búsqueda $y$ en el reconocimiento implicico de que el hombre no está fundado en sí mismo, en el fonćo, se coinci de en referir nuestra realidad a la Realidad que 11 amamos Trascenden cia. Dios, o como se quiera llamar. En eso coincide cualquier creyen te de cualquiera religibr.

Un paso más se da, por ejemplo, cuando el creyente cristiano estina que posee razones poderosas para convencerse de que por la forma de su propia tradición tistórica, formada por la tradición biblica y por su propia cultura y ubicación, el cristianismo es el acceso o intento de acceder a Dios nés cercano a la realidad objetiva de Dios que el ofrecido por otras tradiciones religicsas.

¿Cómo se puede postular eso? En la medida en que, en una perspectiva de historio religiosa comparada, sea posible mostrar que el aporte o respuesta dados alproblema del hambre por la tradi ción biblica-cristiana es mús genial que el que dan otras tradicio nes. Entiendo por genial, como ya lo dije, que es un "botb́n" que ajusta mejor al "ojal"; corresponde a un mejor diagnóstico antropológico de cual es el problema y toca mejor ese problema.

\section{HINOUISMO VEDICO}

El Hinduismo en su origen $y$ en su permanencia se ubica en el continente de la India. Noce o orillas del río Indo (en persa Sindi), en lo que se llama el Penyab, noroeste de la India. EI Hinduismo se 1 lama en terminología hindú el Samatana-Dharma: la doctrina del desapego. Este Hinduismo tiene dos rálces ya que es fruto de un mestizaje, originado de la siguiente manera: Hubo una serie de tribus poco conocidas llamadas hoy día "indoeuropeos" o "arios". Es- 
tas tribus remontan al año 3.000 a.C. Eran de cultura sedentaria, pe ro por alguna razón, alrededor del tercer milenio, empezaron grandes emigraciones, en todas direcciones, desde lo que es actualmente el norte de Alemania (el Beltico). Áń estaba la cuna indoeuropea. Estos grupos tenían su propia cultura elaborada, su organización social y su idioma. Y se fueron desparramando a traves de los siglos, por supuesto, por tribus que, provenientes de lo misma cuna, se fueron aislando unas de otras. Algunas de ellas llegaron al sur de Europa y fueron en gran parte, el fundamento y germen de lo que sería más tarde la cultura grecorromana. Otras llegaron al Asia Meror. A Palestina, por ejemplo, 1 legaron los hititas que son indoeuropeos.

Llegaron también a Mesopotania, y tuvieron all1 un gran imperio: fueron los hurritas. Otros grandes grupos 1 legaron más al este y alcanzaron hasta el Indo donde establecieron su propia cultu ra, después de un período más o menos largo de tremendas guerras con los autb́ctonos "drávidas". Al final, despues de muchos siglos o a lo menos decenios de luchas, vencieron en el $80 \%$. Fue un proceso un poco al estilo de lo que pasb con la colonia portuguesa, española y anglosajona con respecto a América: se impuso la cultura de los invaso res en un $80 \%$, asimilando un porcentaje de un $20 \%$, autóctono. Algo similar pasó con esta cultura drávida. Muchos se fueron hacia el sur; por eso cuanto más se baja en la India, la tez es más morena, más indígena y cuanto más al norte, más el mestizaje tiende al blanco.

Asf pues, a nivel racial, o nivel cultural y a nivel reli gioso, el Hinduismo es el producto de ese mestizaje, en un gran por centaje producto de lo que era la cultura indoeuropea.

Es en este momento, entre el 1.800 y el 1.400, cuando lo cultura ya se ha sedentarizado alrededor del Indo e incluso empiezan a aparecer textos. Los textos fundamentales son los 11 cmados Vedas. Hay un Veda principal, el RigVeda ("rig" significa verso: el Veda de los versos). Hay ademós otros tres: el Yajurveda, el Somaveda (o Samaveda) y el Atharveda,.. que son comentarios al Rigveda. El Yajur veda contiene comentarios de tipo escolar, por decirlo asf, el Somave da, de tipo litúrgico, con anotaciones musicales y pora uso cultudi; el Atharveda es de carácter más bien mágico: el poder de las palabras de este Veda es utilizado para lograr mógicamente cietos efectos. Por eso es el mós marginado.

El Rigveda es simplemente una compilación de versos, de himnos a divinidades, no más ique eso. La teología védica hay que sa carla de esos himnos: lo que se dice, los elogios que se dedican a tal divinidad permiten ir adivinando el significado del rol que ella juega en lo fe y se puede armar así la tealogia vedica. Pero no hay textos de teología vedica, sino himnos. Muchos de ellos respiran un climo todavio guerrero en que se habla de los enemigos, y a los eneml gos se los pinta como a los indígenas. As 1 se ve que la mano princi $=$ 
pal de estos himnos es la de los invasores.

\&Que podríamos destacar de la estructura religioso de es te primer nivel del Hinduismo?

En primer lugar, los dioses: Los dioses tienen un tremen do paralelo con la estructura de divinidades greco-romanas, con lá estructura de divinidodes persas, y con lo de divinidades mesopotá micas. La razón es muy simple: la estructuración viene de la mismo cuna. Hoy los historladores están de acuerdo que la estructuración original era indoeuropea. Lo fundamental de esas divinidades es la mitificación de la experiencia básica del ser humano en el mundo, que es el cielo, la tierra y lo que hay entremedio, la atmósfera, sobre todo el trueno. Lo que mós les llamaba la atención de la atmbsfera era al rayo seguido del trueno. En la tradición védica el Dios principal es, pues, Indro, dios del trueno. En la tradición griega sera Zeus, el Dios del royo, etc.

Asi, la tríada suprema resulta de la mitificación de la experiencia de la bóveda celeste, de la experiencia de la tierra, sobre todo en su fecundidad (la madre tierra); y de la experiencia de lo que son los feriómenos atmosféricos, el aire que se respira y al rayo, que es lo que mós llama la atención. Por eso hay muchas divinidades atmosfericas importantes o intermedias: genios, malos y buenos que están por los aires, el cielo mitificado y divinidodes subal ternas al dios celeste, la luna -sobre todo-, estrellas y sianos deI zodíaco. Todo se mitifica en divinidades astrales y la tierra con todos sus fenómenos.

Eso explica la similitud que existe incluso a nivel del idioma. Es curioso que en el sánscrito de los Vedas, el dios del cielo se llama Dyaus-pitár (Dios-padre). "Dyaus" equivale al latín "Deus" y "pitór" (podre) es sinónimo de "pater" en latín y griego, de "fadar" en gótico ("vater") en alenínl, etc. Esto indica. que todo procede del mismo tronco lingülstico, el "indoeuropeo", estudiado y reconstruido o partir de los trabajos de F. Bopp, en 1816. Se puede decir que el idiomo indoeuropeo primitivo (o "indogermánico") es el padre de un grupo de idiomas tales como el iranio, al sanscrito, el hitita, al griego, el latín, el celta, el germánico, etc.. etc.

En segundo lugar, el Hinduismo -aparte de esas divinidades elaboradas en la forma antes dicha- tiene mediaciones para relacionarse con esos poderes sagrados, o seo, el culto. El culto estó centrado básicamento en el fuego. Es un elemento "fundamental. Hay un dios muy importante en el vedismo que es Agni y corresponde al fuego mitificado (la palabra sónscrito "agni" significa fuego al igual que la latina "ignis"). El fuego es el mediador a traves del cual se hace el sacrificio a los dioses. El dios Agni es muy cele brado en los himnos védicos donde se les describe, tanto por sus epi 
fanfas (manifestaciones) místicas como por sus atributos divinos especificos. En el fuego se consume un elemento sagrado, el soma,plan ta aromática especial que siempre esté presente en los rituales. Sé la mitifica convirtiéndola en el dics Soma, que ocupa un lugar muy destacado en el panteón vedico por el número de himnos que se le han dedicado. La planta soma es muy rica en virtudes milagrosus; se que ma en el fuego y con el aroma que desprende llega la plegaria, el himno, a la divinidad a la cual se dirige (1).

El fuego es tambien muy importante en Irán y en toda 10 religión persa. Asimismo en las tradiciones greco-romanes. Los dioses "lares" estaban vinculados al fuego, al Lar (hogar doméstico). Incluso nosotros traducimos "lor" por fogón, chimenea. Es el medio por el cual se accede a esa divinidad.

Finalmente, después del aspecto cultual estó la organiza icón social. El Hinduismo es conocido por su muy marcoda estratificā ción en castas. ACuales son esas castas? Fundamentalmente son tres para los descendientes de los invasores orios (arya-varna).

1. La casta de les brahamanes (de Brahma, que es el nombre del Absolutol o sacerdotal. A los brahmenes corresponde el estudio, la enseñanza, la elecución de los ritos sacrificiales, la caridad y el recibir ofrendas personales. En otras palabras, son los encargados de lo cultual que les permite asegurar que las divinidodes están en buena disposición para con la scciedad. Son los mediadores con esa divinidad; también protegen a la sociedad de posibles enfados de Indra o de quien sea; son mediadores para lograr lluvias, fertilidad, etc.

2. La de los ksatiyas es la casta de los nobles guerre ros. Generalmente, en la antigüedad, noble y guerre ro estaban bastante vinculados.

3. La casta de los vaicas de comerciantes y agricultores. Equivale a la 1 lamada "tercera clase". Es la gente que hace andar al pais; no es dirigente pero si importante en la economia $y$ en influjos.

Estas tres castas principales estaban rigidamente jerarquizadas en el vedismo y tenían sus derechos: por ejemplo, eran las únicas que podian acceder al texto de los Vedas.

Aparte de las nombradas, hay una cuarta casta que es la de los sudras, formada por pequeños comerciantes, campesinos y todos los quo hacen 105 mós humildes trabajos manuales ( $e l$ pueblo bojo). Se compone de un amasijo de población no oria, aunque integrado en los cuadros arios. Son considerados intocobles por lo que se refiere al socrificio, pues participaban en Este solo indirectamen te. Pero esta casta cada vez estó mós integrada al conjunto de las 
otras tres.

Finalmente estón los parias, que son los sin clase, la gente que existe para servir a las castas. Ese es el papel de su existencio. No pertenecen a uno casta sino que estün en función de esas castas.

La estratificución estaba consagrado por la visión social del Hinduismo vedico. Esto proviene ya, sin duda, desdo el nor te de Europa y lo encontramos con variantes, por ejemplo, en la esclavitud del período greco-romano, en el Irä́n, etc. Pero, donde mós fuertemente se elaboró y con mós teologla detrás, fue en lo tra dición hindú. El sistemo se horó más marcodo en el Hinduismo clo sico, del cual hablare a continuación.

\section{HINOUISMO CLASICO}

El punto de partida del 1 lamado Hinduismo clásico sur gis de la clase sacerdotal, de los brahmanes, y se caracterizo por una mayor elaboración teolbgica de los textos de los Vedas. La casto sacerdotal fue tomando cada vez mayor preponderancio. Los brahma nes (o brahmines), dedicados "full time" a la exégesis de los Vedás expusieron sus grandes comentarios en los libros llamados Brahmanas. escritos en un periodo que va desde los siglos VIII al VI Ty sigue algo despues). Todo Veda posee su Brahmana: El Rigueda (o Veda ori ginall tiene el Aitareya, el Yajurveda tiene el Cataphata-Brahmo na; $y$ en general el Somaveda (o Samaveda), que es sobre todo cul tual y objeto de muchos comentarios brahmánicos.

ZQue se podrla señalar de eso elaboración teolbgica brahmánica dentro del Hinduismo clásico?

En primer lugar, algo muy importante en relación a la concepción de la divinidad. En el Hinduismo vedico onterior, las di vinidades son todas producto de mitificaciones, a las que correspoñ dería un papel cósmico o social, Dyaus-pitár es el dios del cielo, Prithivi es la dioso-tierra, $y$, sobre todo, Indra, dios del trueno, de la atmósfera y del relámpago, es el mós popular len el Rigveda so le dedican unos 250 himnos), el que tiene más ingerencia, el mós temido y venerado. En cambio, la bbveda celeste está muy tranquila allá arriba. Se adoran y son objeto de culto las divinidades astra les como la luna mitificada, las estrellas y los signos del zodra co. Sin embargo, la boveda en si, el dios-celeste, el ser supremo, se va "alejando" de los hombres, haciondose inaccesible. Este es un fenomeno que se observa en muchas religiones; el dios supremo se convierte en un "dios ocioso" ("deus otiosus"), en un dios "inútil", al cual ya no se recurre. De alls que haya poca magia vinculada a e1. Correlativamente, los dioses secundarios, los que desempeñan el papel cosmico, mucha importancia en la espiritualidad y un cul- 
to muy rico, con mucho recurso mágico.

Asl, pues, ese dios supremo tiene poco importancia; es decir, no la tiene para la elaboración metafisica de la divinidad. Pero si la tienen, en alto grado, los "dioses útiles...".

Ho aqu1, por ejemplo, algunas estrofas de uno de los himnos del Rigveda dedicados a Indra: Observemos lo que se le atri buye a Indra, que teologla hay detrós de ese recurso a Indra. Dí ce asf:

"Aquel que recién nacido es el primero de los dioses" (2).

"Aquel que tiene el honor de todos los dioses por sus grandes maravillas,

Aquel cuyo poder es objeto de temor para el Cielo y pa ro la Tierra,

Aquel que hace reconocer la grandeza de su fuerza;

Sabed, oh mortales, que ese es Indra.

Aquel que ha fijado la Tierro en su curso, que ha apaciguado los montoñas agi tadas,

Aquel que ha extendido el firmamento espacioso,

Aquel que ha consolidado el Cielo:

Sabed, oh mortales, que ese es Indra.

Aquel que habiendo destruido a Ahi (3), ha puesto en libertad los siete rlos,

Aquel que ha recuperado las vacas retenidas por Vala

(4), que ha engendrado el fuego en las nubas,

Aquel que es invencible on los combates:

Sabed, oh mortales, que ese es Indra.

Aquel sin el cual los hombres no pueden triunfar,

Aquel de quien invocan el apoyo cuando están

envueltos en los combates,

Aquel que es prototipo del Universo, que

vence al enemigo y que nunca retrocede:

Sabed, oh mortales, que ese es Indra" (5).

En el fondo, se atribuyan a Indra las categorías do Creador, de Omnipotencia, de Protector, etc.

En el Hinduismo clásico, a diferencia del védico, la reflexión brahmónica gira ya alrededor de la categorla de Absolu to. No de la de divinidades jararquizadas -que se mantienen, y por lo tanto de un politeismo jerarquizado- sino que más allo do ellas, del Absoluto. Esta categoría estó ya alguna vez indicada en los Vedas con el nombre de Brahma, pero sin darle mayor impor tancia.

Brahma, en el brahmanismo, es pues, igual al Absoluto, 
una categoría transdivina. No es un dios mós, porque el dios del cielo es Dyauspitár, Indra es el del trueno, etc., etc. Brahma es el Absoluto, el que no ha nacido, el que tiene las categorías propias de Dios. Está fuera del ciclo de reencarnaciones. El que no ha empezado a existir. Brahma se convierte en el punto central de referencia de la espiritualidad brahménica y del Hinduismo que va desarrollóndose alrededor de eso espiritualidad.

Ahora, para vincularlo con la tradición vedica ante rior, hay tres nombres que se atribuyen a Brahma. Es una especie de "trinidad" hindú que se llama trimurti. Equivale a la elaboración de la categorla teológica de trinidad: un solo Absoluto con tres nombres: Brahma es el primer nombre, los otros son Siva y Visnú. En la tradición hindú (como en lo cristiana) figura tam.bi Gn el símbolo del triángu lo. Además hay otra manera de simboli zarla: un rostro con tres caras; una mira hacia el frente $y$ las otras dos a cada lado, pero tienen un solo cuello; es un solo tron co con tres caras.

¿Que simbología metafísica hay detrás? Hay una reflexión bien interesante de corte dialectico; como la occidental hege liana. También la encontramos aquí es la relación dialectica de la nagatividod, la positividad y la sintesis. A Visnú se le atribuye $\in l$ polo positivo y por eso Visnú adopta a veces la forma de miel que simboliza la cohesión. Siva es lo negativo, absolutiza do, divinizodo. Y lo que en la historia de los roligiones se 1lama la "coincidentla oppositorum" o el misterio de la totalidad. el Absoluto en quien coinciden las contradicciones, eso es Brahma.

Tanto Siva como Visnú tienen lo que ya la tradición brahménica elabora: los avatares o avataras, que son las encarna ciones. Brahma no lo hace. Es decir; siendo nombres del Absoluto, toman formas diversos, se historicizan. Asf, por ejemplo, como ve remos al final. Krishna es un personaje religioso considerado co mo la octava encarnación de Visnú. En la elaboración mítica de las diversas encarnaciones hay toda una especulación rayana en la fantasía. Pero todo esto es el mundo del Absoluto, que no tiene nada que ver con los dioses védicos, que siguen funcionando como dioses que no son lo Absoluto, estón colocados en el ciclo de las reencarnaciones inmanentes del cielo para abajo, del cosmos. Y estos dioses pueden de alguna manera por infidelidad, a su momento encarnatorio, descender a otro mundo, dejando de ser dios. Y al re vés, el hombre puede ascender y reencarnarse en forma divina, siñ que eso sea todavio estar en lo Absoluto. Esto es una innovación teologica propia del Hinduismo clesico en lo que toca a los dioses.

El otro aspecto que quería señalar, de la elaboración teológica del Hinduismo brahmónico, es justamente la categoría de reencarnación, pero ya estructurada. EEn que consiste? Es un tema que tocaremos también al referirnos al Budismo, al Krishnaismo y a todas las formas relacionadas con el Hinduismo. 
La tradición religiosa hindé ve el tiempo en foma cícli ca. Pero mús que del tiempo hay que hotilar de la existencia. Lá existencia, en versión hindi, es lo que lloman el sumeara la veces tambien, maya) (6). Samsara es propianente el ciclo de las reencarnaciones. "Todo lo que existe ha existido y existira". Termina una forma de existencia para comenzar otra, disi jnfinitamente. El ciclo de reencarnociones corresponde a la existencia de todo lo que no es Io Absoluto, el cual, en este sentido, no posee el atributo de existencia. El Absoluto es lo que no existe.

En este momento avanzamos una teorla que nos sorprende: en el Budismo oiremos heblor del Nirvana como iqual a "la no exis'cen cia", a la extinción total de los condiciones del ser, el "estado del fuego apagado de un soplo". La "no existencia" con respecto a la existencia es una categoría que tambien se encuertira en el Hin dufsmo clásico. Es decir: si por existencia entendemos al existir, Dios no existe, Diso es la negación de la existencia: Todo lo que existe, incluidos los dioses que no son lo Absoluto, forman parte de este ciclo de reancarnaciones, sucesión ritmica de creaclones y destrucciones cósmicas, que se ha elaborado en mucha literotura en la forma siguiente:

Cada ciclo comricto o mohayuga ("maha" significa grande) se compone de cuatro unidodes, do ciclos mús pequeños 11 amados yugas. Asf hay un primer yuge, un segundo, un tercero y un cuarto yuga. Al terminar un ciclo completo sa produce una "dzsolución" o pralaya, que es una especie de $f$ in del mundo parcial, como una hecatombe que do fin a una cantidad de encarnaciones y a un recomenzar en otros formas de encarnación. Al final de un cierto nümero de mahayugas, que es variable según las diversas escuelas (por ejemplo, mil para el Bhagavad-gita); viene el mahapraleya... (gran pralaya) gue es un fin del mundo más radical y continúa en un nuevo proceso, ro tando asi indefinidamente. Hay escuelas que fijan la duración tempo ral de estos ciclos cósmicos en seis millones de años, otras en 4.320.000 años; pero todas son especulaciones. Y en este ciclo estamos todos, desde las piedras hasto los dioses, girando. Cuando ter mina una forma de encarnación se suceden otres. Ellas son mús o me nos prolongadas depandiendo de la forma de 'existencia que se tenga en el momento. Así, una piedra dura mśs, hasta que surgen muta ciones químicas adecuadas que las disuelven y recomienzan en otra forma.

Lo mismo ocurre con los vivientes: al terminar una exis tencia, no dejamos de existir sino que cambiamos de modo de existir. nos reencarnamos en otra forma. A eso va vinculada una categoría de juicio. Vale decir, la forma en que nos reencarnamos depende del "comportamiento" más o menos adecuado a lo que se espera de la forma de existencia que uno tiene. Cuanto mes correcto sea, mós se pue de ascender en una encarnación ulterior a un modo superior de exis = tencia. Si no se ha respondido de acuerdo con lo esperado se puede descender a nivel inferior. De modo que un dios puede dejar de ser 
dios y un hombre elevarse a la categoría de dios o por el contrario, pasar a ser serpiente. O una piedro pasor a ser viva, o un animal reencarnarse en farma de hombre. Adeniás, hay otro punto imporiante dentro del sistema de castas de los hindúes: un poria puede ascender y reencarnarse como brahman y a la inversa, un brahman bajar a pa ria. La estructuración en castas, a nosotras nos choca enormemente como problemo social. A ellos no, porque hay una relatividad comple ta del momento actual. Si el paria es un buen paria, tiene la oportúnidod de llegar a ser brahman, por lo que no le da importancia a la encarnaciśn actual, y un brahman puedo dejar de serlo. El estado en que se encuentran lo relativizan mucho más que en nuestra socie dad, donde absolutizomos més nuestra existencia concreta. Esta postura es importante para no proyector nuestro visión y entender su ma nera de enfrentar la existencia.

Otra categoría teológica importante es la de Korma o de residuo kármico. El Karma es el responsable de que yo siga existien do. El Karma es el apago que un existente tiene a la existencia. Wientras un existente esté apegado a la existencia, de alguna manera la desee, tiene Karma y si al terminar esa existencia no ha eliminado completamente ese apego, se reencarna. Es una especie de "pecado original" en el Hinduismo. Es un residuo que me viene de la existen cia anterior, porquela termine sin haber aniquilado el aepgo a la existencia. El que yo me encuentre existiendo quiere decir que ter miné la existencia anterior todovía con Karma, con apego, y eso de= termind que renaciera en otra forma. Esa perepectiva la veremos sobre todo en el Budismo y será muy imporiante para elaborar la teoría y la Etica budista.

El problema es entonces cómo eliminar el Karma de tal manera que al terminar la existencia ya no hayo Karma y en consecuen cia no haya reencarnación sino cese de existir. Eso es la salvación.

Este problema ya esto en el Hinduismo, pero el Budismo lo elaborará más a fondo y més radicalmente. dos niveles:

El Atman (7) ses otra cctegorla de importancia. Tiene

a) Lo que se llama el Maha-atman, (gran Atman), que es la categoría del Absoluto hecho inmonente, es decir, es el Alma del mundo, el Alma de lo existente. Es una categoría metafísica para decir que todo lo que es, es en el fondo Uno. La multiplicidad, por lo tan to, es signo de no ser; cuanto mós se es, más se unifica, más sē encuentra el fondo unitario de la realidad; por eso Dios es Uno, es el fondo que unifica todo lo que es. La multiplicidad es siempre de superficie.

El concepto de Maha-atman correspande a una metafísica propia de la existencia para decir lo mismo que dice, en términos 
teolbgicos, la palabra Brahma. En el fondo el Maha-atman y Brahma es como distinguir el Ser como categorfa metafísica y Dios. El Ser es el Uno y eso teológicamente se llama Dios. Aunque filosoficamen te se llame el Ser.

b) Distinto del Maha-atman es lo que llaman el Jiv-atman, pequeño ("jiv" es pequeño), que axactamente es alma individual, atiribuida, no sólo a los vivientes humanos, sino a todo lo existente indivi dualizado: una piedra tiane su almo. En el fondo, es el prihcipio de la individualidad, es el ser en cuanto individudizedo. Este Jiv-atman es el que se reencarna. Es como una energía en potencio que se distribuye en millones de salidas. Esa salida termina su función y la energía es absorbida hacia su fondo común y se distri buye por otro lado, sole de otra manera. Ahora, todos esas indi = vidualizaciones de Jiv-atman buscan volver al Maha-atman, es decir, reducirse al Uno y dejar la multiplicidod.

Podemos ver, entonces, que al mundo del Hinduismo nues tra tradición biblico-cristiana de Dios como persona, de nuestros valores de la personalidad, no le dice nada. Es decir, todo lo perso nal es signo de no tener valor; hay que despersonalizarse, suprimir la individualidad, volver al "anonimato" del Maha-atman, a eza espe cie de fondo común de Moha-atman. Se considera la individualización de la conciencio en multiplicidad más como un defecto que como un valor; se ve como un no ser más quje como ser. Entonces su sistema de valores: se ordena justamente a eliminar esa individualidad múlti ple. El ideal es reducir todo al Uno indiferenciado. Es una perspec tiva muy distinta a la de nuestra tradición.

Todo esto puede parecer al interior de nuestro sistema como cosas de otro mundo. Basta sin emborgo, leer algún místico cris tiano para ver en su lenguaje una cierta tendencia a perder su iden tidad propia, ser uno con Dios. San Pablo llega a decir: "Ya no soy yo, quien vive; es Cristo quien vive en mi". Hay una especie de bús queda mistica de perder la propia personalidad en función de la única personalidad que interesa. En la perspectiva mistica, surge mucho ese buscar perderse uno en el 0tro, que es el Ser. No por la perdida sino por el "Otro". Esta pista, pues, no es tan ajena a nuestra tradición religioso.

\section{EL BUDISIMO Y SUS DERIVACIONES}

fundamentalmente.

Voy a pasar ahora a lo que ocurre en el siglo VI a.C.

Lo tradición del Hinduismo clesico que empezó hacia el siglo octavo, estaba en su plenitud en el siglo VI a.C. Ahr empiezan ya las elaboraciones teológicas geniales, que comienzan a hacer escue la por su cuenta. En ese contexto hay que ubicar a Buda. Buda toma su propia perspectivo del Hinduismo elaborado por lo trodición brahmá 
nica. Asl nacen en el siglo sexto el Budismo y otras corrientes: Hay uno que viene de antes, pero que tomarós especial fuerza y asumiró ele mentos del Budismo: es el Krishncismo.

\section{a) ¿Que podrlamos decir del Budismo?}

Primeramente algo ya insinuado al principio: El siglo VI a.C. es el siglo de oro de la historia religiosa de lo humanidad. Tombién empiezo en ese siglo el milenio de oro, que va desde el siglo VI a.C. al siglo VI d.C. Coinciden curiosamente en 6l, en diversos lugares, grandes movimientos religiosos. Tenemos el Budismo en la In dia. Simultáneumente en Persia estó la reforma zoroástrica. Tambieñ es el periodo más importante del profetismo biblico: En la historio de Israel, el segundo Isaĺas, Jeremfas, Ezequilel, son de esa época. Las dos grandes 1 íneas contemporáneas de la tradición China, la de Confucio y la del Tooĺsmo de Lao Tse, son tambien del siglo VI a. C. Todo eso es simultóneo, es un proceso enormemente rico. En el siglo I nace el Cristianismo. Por Ultimo, en el siglo VI d.C. emerge el Ís lam. Es un milenio realmente fuerte. Despues de ese periodo ha habi= do movimientos chicos, sin la envergadura que tuvieron aquellos que marcaron a millones de gente de la misma manera. Es un período, en ese sentido, de oro en la historia religiosa de la humanidad.

\section{El Budismo nace en ese contexto realmente notable.}

¿Qué se puede destacar del Budismo? En primer lugar a Buda que es un personaje poco conocido por una razón muy simple: Tuvo la suerte de estar en un continente poco interesado por la objetivi dad histórica, a diferencia de la cultura occidental, en donde hay historiadores preocupados de buscar tumbas vacias, o si se dijo o no tal cosa, etc. En la tradición budisto, todo funciona con mayor "bue na fe". "Entonces se hace muy dificil distinguir 10 mitico de lo histórico, porque se transmiten ambas cosas con la misma validez. Hay menos capacidad critica, o menos interes critico de discernir las fuentes, etc. Los que se han interesado por eso han sido los historiadores occidentales. Y se han encontrado con un mundo imposible de manejar. Buda es histórico, y es tambien mitico. Y hay tremendas obras dedicadas a $€ 1$.

Lo que parece cierto es que nacio y murib en el norte de 1a India, alrededor de Benares. A esa ciudad sagrado iba muy segui do. Su rol fue como el de Jerusalén en lo tradición palestino de Jesús. Era un hombre que tuvo discipulos. Se sabe, según todas las tradiciones, que eran cinco; predicaba y vagaba con sus discipulos de principado en principado (8). En algunos penetrb fuertemente, en otros fue expulsado. Tuvo peleas a muerte con la casta brahmánica -al estilo de Jesús con los fariseos-, que inicialmente se lo quiso ganar. Buda fue combatiod y tuvo que huir. Despues de muerto 61 (muere de muerte natural, parece que de viejo), sus discípulos empiezan a tener problemas. Son perseguidos y el Budismo tiene finalmen- 
te que huir de lo India, y cobijarse en la cordillera tibetana del norte -donde emergeró después la variante del Dalailama-, y en la isla de Ceilón, olejada del continente. Los dos focos de Budismo de la India, no están pues, en el continente: Se calcula que hoy día no hay más do un millón de budistas en lá India, y los que hay están so bre todo concentrados en Ceilón y en el Tibet.

El Budismo resultó fundamentalmente una religión de exportación. Un poco al estilo del Cristianismo (en Palestina hay pocos cristianos comparativamente; hay mós judios y musulmanes). Todo el sudeste asid́tico es budista: Camboya, Laos, Vietnam, etc.' Ahí es donde está el grueso. Hay también millones de chinos budistas aunque con el mestizaje de las tradiciones propias, sobre todo taols tas. Algo similar ocurre en Japón, en menor grado.

¿Que aspectos destacoría de lo que Buda pretendió, de su mensaje? Distinguiría tres cosas: Un primer aspecto social. Buda es un reformador social, sin pretenderlo directamente; lo es "de posoda". Al estilo de Jesús, que tampoco pretendió serlo, pero, "de pasada", su mensaje plantea un problema social. Se puede derivar una "Doctrina Social de la Iglesia", sin que encontremos en la inter vención de Jesús un interés social propiamente directo. Con Buda ocurre exactamente lo mismo: a nivel sucial, debido a la perspectiva de su mensaje, a su forma de ver y de comprender el Hinduismo estaba en contra de la estratificación : en castas. Eso lo convierte en subversivo para "el status-quo" que viene de la trodición hindú. Y lo es porque, como dije antes, los Vedas eran sblo accesibles a las cas tas arias, o sea a las tres primeras. Buda rompe ese esquema, y di= ce que cualquier persona puede entrar en el "camino de la ilumina ción" (según su propia nomenclatura), sea paria, sea de otra casta. Sus biografías cuentan que tuvo un hijo, pero llegado el momento de su primera iluminación, donde descubre la verdad, llega al acuerdo con su mujer de separarse, y se va a la selva. A su hijo lo deja pa ra que después también siga su vida.

Buda era de casta noble, del clan de los sakias, y cuando le plantearon, de acuerdo con el sistema hinde, que le toca ya elegir mujer, la cual por supuesto tenf́a que ser de su propía casta y no de otra, él declaró que no le importaba la casta si posue determinadas cualidades, todas de tipo moral.

Se ve en la tradición que Buda tiene una indiferencio por la estratificación; la relativiza completamente, formula criti cas muy fuertes con lo cual marca una especie de ruptura. Hoy textos conservados atribuidos a 61 en contra de los brahmones. De hecho, 61 aceptó todas las castas, pero llega un momento en que los seguidores budistas no aceptaron brahmanes: la pugna se hablo hecho tan fuerte que ya no se pudieron tolerar. 
Uno segunda perspectiva del Budismo es la reforma pro plamente religiosa. Algunos historiadores hablon del Budismo como una religión "atea". Y reaimente Buda nunca habla de Dios. En los brahmanas hay elaboraciones sobre los himnos védicos que Buda simple mente se salta. No existe la categoría de plegario en Buda; no hoy dioses a quien recurrir, ni se interesa por ellos. Quien se acerco con la categorta tradicional de los dioses a mirar el Budismo original, se encuentro que folto el capitulo principal: No hay dioses, no hay plegarias, no hay consejo de rezar nada. Los monjes budistas originales no rezan, meditan; no establecen contacto con seres trascendentes.

El tercer ospecto es lo ética; es decir, que es lo que hay que hacer en la existencia en que uno se encuentra.

La ética es lo fundamental y eso es lo que elabora el Budismo. No lo hace a nivel de teología propiamente tal. No elabora noda sobre estructuración social, s6lo se salta la estructura que existe, no dóndole importancia. No se preocupa de los dioses védi $\cos$.

En cambio todo su culto es la dimensión etica de lo existencia. A eso apunta y es lo inico que interesa al Budismo. Por eso, según algunos, se trata propiamente de una Ética y no de uno re ligion.

Hay miles de textos atribuidos a Buda. Después de su muerte y a lo largo de unos seis siglos, sus discípulos se reunieron para realizar lo que nosotros llamarlamos "concilios", con el ableto de fijar cuóles textos representabon más fielmente lo doctrino de Bu da. En esa forma se fue confeccionando el 1 lamodo canon pali. que contiene la doctrina budista ortodoxa. Se le denominb "pali", porque las obras que contiens estón escritas en el dialecto "pali", derivado del sánscrito que constituya el lenguaje literario de los budistas del sur. Probablemente fue el habla convencional de las clases altas en Kosala, al noreste de la India, zona donde nacis Buda (9).

El canon pali reone lo más importante y deja fuera estu dios muy numerosos, considerados no ortodoxos. De este canon pali, conlunto que fue llamodo Tripitaka ("Triple Cesto") quisiera presen tor un trozo del texto denominado Udana, de la llamada "primera Ilu= minación" de Buda.

Cuentan que Buda fue a un bosque (bosque es el equiva. lente a nuestro desiertol para buscar la soledad y meditar. Bajo un arbol, en una postura especial, cómoda (en "palanca" que sera la pos tura "Yogui") para poder abstraerse de todo, empieza a descubrir 10 substancial, el meollo de la existencia.

El título del texto es "paticchasamuppada" en pali lpo 
títyasamutpada, en sónscritol que significa "generación condiciona da". Dice así:

"Cuando Esto existe se produce aquéllo. Cuando surge és to surge aquéllo. A saber: los residuos kármicos surgen teniendo como causa determinante a la ignorancia. La conciencia surge teniendo como cousa determinante a los residuos kórmicos. La individualidad surge teniendo como causa determinante a la conciencia. Los seis sen tidos (10) surgen teniendo como causa determinante a la individuali= dad.

El contacto surge teniendo como causa determinante los sentidos. El deseo surge teniendo como causa determinante el contac to. El apego surge teniendo como causa determinante al deseo. Lá existencia surge reniendo como causa determinante al apego. El nacimiento surge teniendo como causa determinante a la existencia. Lo ve jez y la muerte, la pena y el 1lanto, el sufrimiento, el desagradoy la inquiletud, surgen teniendo como causa determinante al nacimiento. Así se produce todo el cúmulo de sufrimientos" (11).

Ese es un texto famoso atribuido a Buda. ¿Cuál es su ló gica? ¿Qué hay detrós de Esto? Verifica, en primer lugar, que la existencia es un problema, en el sentido de que no está fundado en sl misma, no se sostiene por si misma, no es un Absoluto. Eso la con vierte en maya; el existir es maya (ilusorio) para Budo. El ciclo de reencarnaciones o samsara es maya. Esto quiere decir que es malo existir, que serla mejor no existir.

Por otro lado verifica que hay mucha gente que suire. Entonces, se pregunta ¿cómo salvarse de la existencia? $Y$ para po der prescribir un buen remedio, tiene que hacer un buen diagnóstico. ¿Que diagnóstico de la existencia hace Buda? En primer lugar, que todo el mal de la existencia viene de la ignorancia, o sea, del no saber, del estado de ser sin conocimiento, que para Budes es la causo de prácticamente todo devenir dinámico de la vića. La ignorancia comprende el móvil ciego de vivir, es cousa de ideas ilusorias, de deseos terrenales, de errores, falsas doctrinas, etc. En resumen, el hombre es producto de la ignorancia y ella hace que quedo apegado - la existencia, que no pueda superarla por no captar ni saber correctamente cómo ubicarse. Por consiguiente, como el primera mal es la ignorancia, el primer remedio seró la búsqueda de la sabiduría co mo categorla correlativa. Por la ignorancia acumulamos Karma y hé aquí la ilación directa: porque yo no sabía -de buena fe,inclusoque algo era malo (por ejemplo que era malo amar a mi esposa, estar apegado afectivamente a mis hijos, etc.), quedo con Karma y sigo existiendo.

De modo que lo primero es estar bien ubicado, saber que es el mal a evitar y quo es lo que hay que buscar. Todo deseo es ma10, produce Karma $y$, por tanto, apego a la existencia. Y como ignorancia, deseo y existencia son interdependientes ello basta para ex- 
plicar la concatenación ininterrumpida de nacimiento, muertes y reencarnociones en el ciclo de samsara.

¿Ese Karma qué hace? Ese rasiduo kármico que tengo qui zás porque no sabía que tenía que desapegarme, produce la concien. Es decir, ahora que yo me planteo el problema, me doy cuento de que tengo el problema de ini conciencia porque existo. Tengo conciencia porque estoy existiendo $y$ estoy existiendo porque tengo Karma. La conciencia en lo que yo me planteo el problema la tengo, -parabien y para mal-, por culpo dol Karma, del apego. Y la conciencia produce la individualidad de los muchos conscientes. Esa individualidad (Jiv-atman individualizado) produce algo asf como ventonos, ya que se trata de muchas individualidades que están en el mundo ambionte. La ventana de nuestra individualidad nos permite abrirnos a las otras individualidades, a la multiplicidad de la existencia, esta constituida por los sentidos y estos producen el contacto. 0 sea, a traves de estos sentidos me contacto con los otros y el contacto pro duce el deseo. El deseo produce apego, que es otra forma de decir Karma; y el apego produce la existencia. Porque tengo Karma existo; si no tuviera apego no me reencornaría más. Y lo existencia produce el nacimiento. Mientras yo este en la existencia del samsara,mientros yo exista oquí, la muerte no me liberoró sino que a la muerte seguira un nacer de otra manera. Y una vez nacido viene todo lo que significa y comporta el tipo de nacimiento en la encarnación empezado. Ese es el ciclo.

Entonces, a partir de la Luz, de la lluminación de Buda, del diagnóstico sobre la verdad de la existencia, $\in$ l Budismo pro pone los métodos para alconzar la libertad perfefta, para abandonar el ciclo del renocer y lograr un estado atemporal, de no-condición, de no-morada, de no-limitación (el Nirvana). Entre ellos figuran ol "Gran Vehículo" (Mahayana) o el "Gran Camino" y el "Pequeño Vehícu 10" (Hinayana) o "Pequeño Camino". El Gron Vehículo es el camino etico donde puede entrar todo el mundo. Tiene dos momentos: El primero es el de la buena voluntad. Una vez que se reconoce la necesidad de superar la ignorancia y llegar a la sabiduría para ubicarse correctamente en la existencia (de lo contrario se corre el riesgo de no poder salir nunca de ellal hay que ser bueno, o sea, tener una actitud buena, evitando el mal y haciendo el bien. Algo similar a lo que expresa Jesús cuando el rico le pregunta: "źqué tengo que hacer para ser perfecto?". Le contesta, "primero cumple los mandamientos". O sea, haz el bien: no mates, no robes, etc., etc. Es la Eti co natural. Es decir, primero, no hogos el mal (lo que es obvio qué us malo sea cual sea la perspectiva etica en que te ubiques).

Uno vez establecido 6sto, viene la meditación inicial. Concentrarse en la Verdad. La verdad es que todo lo que es samsaro oe maya, es malo; por lo tanto, el apego a cualquier realidad existente : perjudica, te aleja del Absoluto. A medida que te concentres en 6sto te irós despegando no sólo de hacer el mal (como matar, ro- 
bar, etc.) sino de cosas que llamamos buenas (como casarse, comer po 110, etc.). Despréndete de todo, deja de lado todo: Si quieres ser perfecto, desapegate; concéntrate en lo único necesario.

Una vez que vayas progresando en el proceso de medita ción, llegaró un momento en que pasarás al "Pequeño Vehículo". Es un camino monástico, estructurado. El Budismo tiende a proyectarse siempre hacia la vida monástico como búsqueda religiosa. Si uno sigue la dinámico propia de lo que es el desapego budista, llego o buscar una organización, una formo de vivir que le permita estructu ralmente estar más desapegado. Por ello lo fuerza del Budismo est $\bar{\delta}$ en los monasterios. La comunidad monústica es una forma que lo ca racteriza mós aún que a la vida monóstica cristiana.

Si deseamos seguir haciendo comparaciones con el Budis mo; hay que destacar que ei cristiahismo no propone para todos 10 mismo. Es decir, que cada persona puede tener un camino diferente para alcanzar la salvoción. Es al concepto del Vaticano II. La santidad se da para todos, los laicos, los sacerdotes, etc.., cada uno con su carisma, puede alcanzarla por su camino propio. En cambio, el Budismo no tiene esa perspectiva; hay un solo camino con diversas etapass Pero todos los que avánzan por lo primera etapo, es decir. los del Gran Vetículo, mientras permanezcan en ella se irén reencarnando. Unicamente los que entran en el Pequeño Vehiculo, -él único que por definición suprime completamente, en su tope, el apego $y$, por lo tanto, el Karma-, solo esos acceden al Absoluto. Ellos alcanzan el Nirvana, la extinción de la existencia, el estado atempo ral, de no-condición, de no-moracia, aespacial, de no-limitación, de Libertad Perfecta. En otras palabras, negativamente, el Nirvana es "extincion". "negación total", "indeterminación indiferancicda". Pe ro evidentemente el Nirvana es algo positivo. Otra cosa es que Buda da haya rechazado darle nombre; no ofrecí ninguna definición. El Nirvana es lo innombrable, lo inefable para nosotros. Su aspecto po sitivo es que constituye "La Libertad Perfecta" y es identico a "La Iluminación Perfecta" (Bodhi). Buda menciona una y otra vez sus "atributos". Los santos liberados (arhats) "han alcanzado una bien aventuranza inquebrantable"; el Nirvano "es beatitud". Es el "noser" con respecto ol ser-en-el-mundo $y$, en ese sentido, es el Ser por antonomasia; pero es un Ser ton infinitamente distanciado de lo que llamomos ser, que es mejor no decirle cor. En el fondo es una categorla casi mistica. Por ella se busca evidentemente acceder a la salvación, liberarse de la existencia del samsara, dejar de existir como ser intramundano. Y a ése Ser, a la hora de asignarle un nombre le llaman "No-Ser", para evitar que alguien imagine al Nirvana con una visión antropomórfica.

\section{b) El Budismo posterior o Buda}

Hablamos anteriormente del canon poli, fruto de 10 necesidad de codificar y de canonizar las doctrinas de Buda. Porque 
desputs de su muerte hablan surgido numerosas discrepancias doctrina les con la formación de sectos (dieciocho en el slglo III a.C.) qué defendían doctrinas cada vez mús divergentes. Con ese objeto se reo lizaron concilios, siendo el mśs destacado el de Potaliputro convocado por decreto del rey Asoko lel tercer concilio según la tradi cibn, hacia el 240 a.C.l. Este rey se convirtió al Budismo, lo propag6 por sus dominios y su hilo ( to hermono menor?) Mahinda lo in trodujo en Ceillín. El conon pali contiene la doctrina budista ortodoxa (Theravada o antigua) que despueśs se llams Hinayanao ("pequeño veh(culo"). Según el Budismo hinayano hay que remitirse a esas ense ñanzas para ser un budista como Buda lo quiso. La salvación era para ol individuo, no para al mundo entero. El ideal era avanzar por el sendero hasta convertirse en santo (arhat, "arhan" en pal1). Por este individualismo, el Hinayana fue llamado también Khadgayana o "Vehículo del Rinoceronte", porque esta animal vive y anda solo. Pe ro este criterio, tradicional, de acverdo con el cual la liberación del samsara śblo puede lograrse por la vida monástica y la renuncia al mundo, se apartaba de la doctrina de la prescindencia del mismo Buda. A partir de esa prescindencia, propia del espíritu mismo del budismo, se elaborb una teología y una doctrina más abierta, libre, más fácil de seguir, que significó una renovación radical del budismo. Este movimiento iniciado hacia finales del siglo I a.C., se denomino Mahayano, literalmente "Gran Vehiculo" en oposicion ol "Pe queño". Por ser el más apto para captar el interós de la gente, pron to se convirtic en la rama mayoritaria del Budismo. En ella entran todos los que quieran, porque por principio, no dogmatizan y todo es posible. Es así como se extendib a la China, al Tibet, a Mongolia, Corea, Japón, etc., países donde se formoron diversas sectas y escue las, adaptóndose a las necesidades religiosas de los diferentes grupos. En Japón, el Budismo mahayana chino llegó procedente de Coroa, en el siglo VI. En el siglo IX se unib a la religín nacional shinto. formándose el ryo-bu-shinto. Mas tarde, el Budismo de la meditación de la "Escuela de la Interiorización de Bodhidharma" (maestro indio que la fundo en China en el siglo VI de C.), fue difundido en el Japón por Myoan Eisai (1141-1215), fundando la secta Zen. Hay varias otras sectas budistas laponesas. Todas las formas mencionadas pertenecen a la escuela Mahayana. El budismo hinayana sólo subsiste en Ceilán, Birmonia y Siam.

Las escuelas mahayanas no estón determinadas por el canon pali, sus obras capitales están escritas en sónscrito. Las pri meras manifestaciones del Mahayana están atestiguadas a fines del siglo I a. C. en los Prajnaparamita-sutras. La literatura es copiosa. El ideal del Budismo Tharavado (o Hinayana) es llegar a la cate goría de arhat o pratyekabudha, individuo solitario que busca su propio Nirvana, o sea, un estado trascendente de liberación, más allá del nacimiento y muerte. En contraste, el Budismo Mahayana, al que sus adeptos 1 lamaron "Sendero de los Boddhisattvas", esteblece como ideal al hombre que pospone indefinidomente su liberación para luchar por la ealvación de todas las crioturas vivientes. A estos 
personajes santos, que repetidos veces renuncion al Nirvana y aceptan voluntariamente la existencia humana, el Budismo Mahayana les confie re la categorfa de boddhisattvos (candidato a Buda). En todas los escuelas budistas se da importancia al Boddhisattva; pero los mahayanistas lo declaran superior al arhat, santo que desarrolla la sabidu rla, pero no en grado suficiente la compasión; no están liberados completamente del "yo" por lo que buscan egoistamente el Nirvana para sí solos. En cambio, los Boddhisattvas, de acuardo con el Prajna paramita, "no pretenden alcanzor su propio Nitvana privado. Por $\in \bar{I}$ contrario, recorrieron el mundo al tamente doloroso de la existencia, $y$, a pesar de ello, deseosos de ganar la iluminación suprema, no tiemblan ante el nacimiento y la muerte. Se pusieron en marcha para bien del mundo, pora la felicidad del mundo, por pledad hacia el mundo...". El Boddhisattvd, aunque perfecto, no entraró en el Nirvana hasta que se hayan salvado todos los seres vivientes.

La escisión mahayónica del Budismo representó el triunfo de una tendencia mós liberal, que modificb y renovb los conceptos, doctrinas y prócticas religiosas. Se marginaron del Budismo tradicio nal Theravada. Mientras que los hinayanas se remiten al patityasamut pada $y$ a todas las formas iniciales clásicos de Buda, los mahaya = nas dejan incluso de remitirse a Buda si es necesario. Uno puede ser budista sin pensar en Buda, porque pensor en Buda puede ser una forma de apego. $Y$, de hecho, la crítica que hacen los mahayonos al Budismo actual es que ha convertido a Buda en un dios; tiene templos y lo introducen en el pantén. Esto para el mahayana constituye lo aberración del mismo espiritu budista. Son budistas sin nombrar nunca a Buda, de la misma manera que Buda era religioso sin mencionar nunca a ningún dios. Lo que les importa es desapegarse. En esa forma elaborando, reflexionando, las escuelas mahayanas fueron desarro 1 lando una enorme producción filossfica y religiosa a lo largo de varios siglos.

\section{c) El Krishnaismo}

El Krishnaismo es uno de los movimientos religiosos de: más importancia del Hinduismo histórico. Su nombre procede de Krishna, un personaje mitificado, que vivió en la tierra, pero que se consideró como la octava reencarnación de Visnú. Vasudevo-Krishna fue un príncipe de la dinastla Vrisni, que habio sido divinizado ya en el siglo $V a . C$. Proclamó uno doctrina ttico-religiosa que fue incorporado posteriormente al Bhagavad-gita, el texto del krishnaismo, su verdadero evangelio. El Bhagavad-gita es un episodio dentro del famoso poema épico el Mohobhorata, la epopeya más extensa de la literatura universal (contiene noventa mil versos). La fecha de com posición del Mahobharata es diflcil de establecer. Toda la epopeyá, tal como ha llegado hasta nosotros, es el resultado de un largo procesa de amplificación, revisión, asimilación y redacción de numero- 
sos relatos, entre los cuales se inserta como tema central "la gran epopeya sobre la lucha de los descendientes de Baharata". Dentro de ella, el Bhagavad-gita viene a ser como el corazón, con Krishna co mo personaje central.

E1 Bhagavad-gita establece la divinidad de Krishna.Es, como ya se ha dicho, la loctuva reencarnación de Visnú, por lo tanto del Absoluto. Es la Suprema Personalidad de Dios Param Brah ma, el Brahman Supremol, que encarnóndose vino a revelar la ver = dad. Nótese que la categoría de encarnación en Siva $y$ en Visnú, no es la misma que corre para los dioses y para los hombres, en el sentido de reencarnación como castigo del Karma. La encarnación de Visnú-Krishna viene a corresponder a la de Cristo en nuestra cate gorfa: Lo hace por los hombres, para que los hombres conozcan la verdad. Viene en función revelatoria, no en función de costigo que tiene Karma y que erd dilos sujeto al ciclo de samsara. Por lo demás, no podía renacer porque es el Absoluto. Krishno desciende para ayudar a los hombres a encontrar la verdad.

¿Que verdad aporta? El tema del Bhagavad-gita entroña la comprensión de cinco verdades básicas: Existe el isvara o con trolador, y los jivas, entidades vivientes que son controladas. Se discute la prakriti (la naturaleza material), el tiempo (la dura ción de la existencia de todo el universo o de las manifestaciones de la naturaleza material) y del karma (la actividad...)

Veamos un capitulo del Bahgavod-gita, que resume muy bien la posición teologica que toma el Krishnaismo. En el capítulo 11. habla Krishna a Arjuna, un personaje que se plantea el proble ma de conciencia antes de una batalla en que deberó mator:

"Lo qua no Es nunca existe, lo que Es nunca es existen te: pero aquellos que ven la verdad conocen la línea divisoria. Pé ro has de saber que aquello que habita todas las cosas es indestruc tible (el alma). Nadie puede destruir a este Uno imperecedero. Es tos cuerpos en los que encarna el Ser Eterno (Atmon) -que es indes tructible insondable- son perecederos. Así fue declarado. Lu = cha, entonces, loh hijo de Bharatal

Quien cree que este Ser mata y quien lo cree muerto, carecen ambos de comprensibn. El Ser no mata ni estó muerto. El Ser no nace, el Ser nunca muere. Nunco su existencio se vuelve existencia. El Ser (alma) es sin origen eterno, imperecedero. Es primordial, no muere cuondo muere el cuerpo... Aquel que conoce a este Ser indestructible y etarno, sin orlgen e imperecedero, źcómo puede ese hombre loh hilo de Prithal, matar a alguien o hacer que alguien sea muerto? Asl como un hombre desecha su vestimento gastada, y se pone una vestimento nueva, así el Atman encarnado, dese cha los cuerpos gastados y penetra en cuerpos nuevos. Las etapos no lo hieren, el fuego no lo quema, el agua no lo moja, el viento 
no lo seca. Es imposible que este Ser sea herldo, que sea quemodo; es imposible que sea mojado, que sea secado. Es eterno, omnipoten te, permanente, inamovible, imperecedero. Se dice que es inmanifestable, que es invisible, inconcebible, inmutable e inalterable. Sobiendo asto, loh el de los brazos poderosos!, no debes lamentar por el..."

$Y$ este versiculo final da la caracteristica clave del Krihsnaismo: "Que tu inter $6 s$ recaiga solamente en la acción, nunca sober sus frutos. Que los frutos de la acción no motiven tus actos, ni permanezcos adherido a lo inacción..."

El resto es una pardfrasis de lo ove tenemos ya en el Hinduismo clásico y en el mismo Budismo: el concepto de la reencarnación $y$, por lo tanto, el desapego con respecto a todas las formas. Con esa base, no importa morir o no morir; es combiar sólo de forma de existencia... Pero en ese último versiculo citado hay un aporte verdaderamente revolucionario: Krishna revela a Arjuna que el "hombre de acción" puede salvarse, sustraerse de las consecuencias de su participación en la vida del mundo, sin dejar de actuar, a condición de renunciar a los frutos de sus actos, de actaur impersonalmen te, sin realizarlos en vista de beneficios o goces para 61 , ni por motivos malos, como el odio, la venganza, sin pasiones. De esa forma, sus actos no engendrarón nuevas potencialidades kármicas.Krish na no rechaza por completo la inactividad brahmánica.

"El abandono y la ejecución del trabajo de uno-diceconducen por igual a la salvación; pero, de las dos aćitudes, la de ejecutár. el trabajo de uno es la mejor".

Y enseña que la suprema inactividad consiste en reali zar actos como si uno los realizara. Existe la ejecución exterior de los actos en combinación con la renunciación interior del mundo. Con la concepción de la acción desinteresada, declara que es preferible la actividad a la inacción:

"Ejecuta tu deber prescrito, pues la accion es mejor que la inacción. Sin el trabajo, un hombre no puede ni siquiera man tener su cuerpo físico...." (cap. III).

De esta manera la tradición vinculada al Hinduismo tiene dos formas de vincularse a la existencia, a pesar de que su meta es llegar al desapego completo. La primera forma es la elaborada póf la situación de Boddhisattva, la cual implica el desapego, pero renunciando al Nirvana hasta que los deinás existentes logren también el desapego. Se trata de una llameda a la solidaridad para con todos los que estén en camino. La otra forma la constituye el Krishnaismo, que incita a la indiferencia en la acción y no de la acción. Precisamente el signo del desapego o indiferencia con res - 
pecto a todo, esté en la seriedad con que realizb la acción que me corresponde elecutar, sea Esta cual fuere. Asl el Krishnaismo permite evitar lo "alienación" ineficiente propia del desapego hindá y budista, 11 amando a la eficiencia activa, sin traicionar la conciencia del carḱcter kármico de la existencia.

NOTAS

(1) Con el zumo de la planta soma se prepara una bebida que provoco una experiencia extática a quien la tama. Ssegún un himno del Rigueda (VIII, 48), el soma es "el guardián de nuestro cuerpo" y "las debilidades, las enfermedades se han puesto en fuga". El soma estimula el pensamiento, reanima el valor del guerrero, aumenta la potencia sexual, cura las enfermedades. Lo beben en común los dioses y los sacerdotes y de este modo se aproximan al cielo y la tierra, se refuerza y prolonga la vida, se asegura la fecundidad" (Mircea Eliade, Historia do las Creencias y de las Ideas Religiosas, I, 227).

(2) Notemos aquí que los dioses también "nacen". Todo lo que no es el "Absoluto" puro y simple, entra en el ciclo de la existen cia, de las reencarnaciones, que rigen tambien para los dioses. Lo onico que queda fuera es la categoria de absoluto, que no es propiamente la categoria de dios. A nivel de dioses, el Hinduismo es politeista, pero a nivel de la reflexión metafisica, es monoteista. Mas, por "dios" se entiende otra cosa.... Indra forma parte del panteón politeista de los dioses, que no son el "Absolutot y por lo tanto ha "nacido" el primero.

(3) "Ahi", serpiente mitica que al despojarse de su vieja piel había adquirido la inmortalidad convirtiśndose en dios.

(4) "Vala", espiritu de las cavernas, es una de las formas de Vitra el dragón gigante, el cual apresa las nubes o vacas celestiales, cuya leche (el agua pluvial) fecunda los sembrados. El mito central de Indra, el mós importante del Rigveda, refie re su combate victorioso contra Indra.

(5) RIG VEDA, himno I. 
(6) Samsara significa "fluir con", atravesar una serie de estados.

(7) Palabra sinserita, que probablemente es de la misma raiz indo europed cile arima (aima); atmen = respirar, en alemän.

(8) En la Gpoca de Buda, la India estaba dividida en pequerios prin cipados o reinos.

(9) El idioma pali se parece al pchlevi, de la tradición irania, que también pertenece a la familia sánscrita. En pahlevi están escritos los comentarios del Avesta, de los cuales se con servan tan s6lo los "gathas", verosímilnente campuestos por Zaratustra (1lamado Zoroastro por los griegos). Ese es el Avesta antiguo, 1 resto del Avesta actual, est compuesto es pecialmente por los libros pahlevis redactados entre los si = glos III y IX d.C.

(10) La teorla budista de la ideación considera que existe un sexto sentido, el manovijñana. Es el central y le atribuye facul tades telepeticas.

(11) Udana. La palabra de Buda, trad. de Carmen Drcfonetti, Barce Iona, Ed. Seix Barral, 1972, pp. 37-40.

El profesor Antonio Bentuc es Dr. en Teologí. Docente de Historio de las Religiones en la Universidad Catolica de Chile. Participo en el Programa de la Facultad de Filosofía, Humanidades y Educación, sobre los Problemas de la Religión. 